\title{
Prevalence and Timing of Tracheostomy and Its Impact on Clinical Outcomes in COVID-19 Pneumonia Patients in Dubai Hospital
}

\author{
Rashid Nadeem ${ }^{a, b} \quad$ Ahmed Najah Zahra ${ }^{a, b} \quad$ Mustafa Hassan ${ }^{a, b} \quad$ Yusuf Parvez \\ Nilesh Gundawarc Mohammed A.M. Hussein ${ }^{a, b}$ Manal Younis ${ }^{a}$, b \\ Manoj P. Mathews ${ }^{d}$ Wasim Khan ${ }^{a, b}$ Majid Ahmed Saleha,b Imran Mumtaz ${ }^{a, b}$ \\ Hebah Rami Aljaghouba,b Ahd Elfatiha,b Alaa Waleed ${ }^{a, b} \quad$ Alaa Ghoneem $^{a, b}$ \\ antensive Care Unit, Dubai Hospital, Dubai Health Authority, Dubai, UAE; bepartment of Neonatology, Dubai \\ Hospital, Dubai Health Authority, Dubai, UAE; 'Department of Pediatrics, Dubai Hospital, Dubai Health Authority,

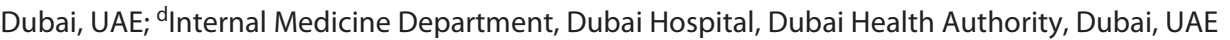

\section{Keywords}

Coronavirus disease 2019 · Tracheostomy · Survival · Length of stay in the intensive care unit · Clinical outcomes

\begin{abstract}
Introduction: Coronavirus has caused more than a million deaths as of October 2020. Hospitals consider tracheostomy after the patient is virus negative, usually after 3 weeks. Prevalence and timing of tracheostomy and its impact on survival among COVID patients are unknown. Methods: A retrospective, single-center study of all patients with COVID-19 ARDS who underwent tracheostomy was conducted. Patients with age $<18$ and patients treated with ECMO were excluded. Duration of ventilation before tracheostomy was recorded. Clinical variables, outcome variables, and confounding variables were recorded and compared between patients with tracheostomy and without tracheostomy. The aim was to determine prevalence and timing of tracheostomy and its impact on clinical outcomes. Results: We found that tracheostomies were performed only in 21 out of 196 patients (10.8\%). Tracheostomies were performed after 3 weeks on average ( $22.1 \pm 7.5$ days). Survival was significant-
\end{abstract}

ly higher in patients who underwent tracheostomy (85.7 vs. $42.5 \%, p=0.001)$. LOSICU was longer for tracheostomy patients than patients without tracheostomy (median [IQR]: 35 [23-47] vs. 15 [9-21], $p=0.001)$. Patients who underwent tracheostomy had a higher proportion of treatment with continuous renal replacement therapy (CRRT) (52 vs. 30\%, $p=0.04)$, more COVID-19 swab testing (6.5 [4.5-8.5] vs. 5 [3-7], $p=0.002)$, more days on mechanical ventilation (34.5 [24-45] vs. 11 [5.5-16.5], $p=0.001)$, and more length of stay in the hospital (54 [38-70] vs. 20 [10.5-29.5], $p=0.001)$. All other factors were not statistically different between the 2 groups. Approximately $29 \%$ of patients had possible falsenegative testing as their swab became positive after being negative. Conclusion: Tracheostomy was performed only in $10 \%$ of our patients with COVID-19 ARDS. Time to tracheostomy was after 3 weeks on average. Survival was better in patients with tracheostomy, but tracheostomized patients stayed longer in the ICU and hospital and utilized more days of mechanical ventilation and CRRT.

(C) 2021 The Author(s)

Published by S. Karger AG, Basel karger@karger.com www.karger.com/dmj

Karger $\stackrel{\text { ' }}{5}$

BOPEN ACCESS
(C) 2021 The Author(s)

Published by S. Karger AG, Basel

This is an Open Access article licensed under the Creative Commons Attribution-NonCommercial-4.0 International License (CC BY-NC) (http://www.karger.com/Services/OpenAccessLicense), applicable to the online version of the article only. Usage and distribution for commercial purposes requires written permission.
Correspondence to:

Rashid Nadeem, nadeem.dr@gmail.com 


\section{Introduction}

Coronavirus has caused more than a million deaths as of October 2020 [1]. Severe infection with coronavirus causes pneumonia leading to acute respiratory distress syndrome and respiratory failure requiring invasive mechanical ventilation. Prolonged invasive mechanical ventilation often leads to ventilator-associated pneumonia and other complications as it is associated with longer use of sedatives, narcotics, muscle paralysis leading to longer need for invasive central catheters, and stationary position of the patient causing line infection and pressure injuries to the skin, respectively. Therefore, tracheostomy is considered for mechanically ventilated patients after 7-10 days if patients are expected to be ventilator dependent for a longer time [2]. Most hospitals consider tracheostomy only after the patient is virus negative since the tracheostomy procedure generates a high aerosol risk of virus transmission [3]. A case series of 14 patients showed that most patients were seronegative after 2 weeks, which suggests it is safer to consider tracheostomy after 2 weeks and seroconversion [4]. Many centers report performing tracheostomy for COVID-19 patients within 14 days [5]. We aim to measure the prevalence of tracheostomy and its timing for COVID-19 patients in our patient population. We compare the outcomes of patients who underwent tracheostomies (28-day survival and length of stay [LOS] in the ICU and hospital).

\section{Methods}

We conductive a retrospective, single-center study of electronic medical records of all consecutive patients with proven COVID-19 infection admitted to Dubai hospital, UAE. We included all patients admitted between January 1, 2020, and June 30, 2020. We only excluded patients $<18$ years of age and those treated with extracorporeal membrane oxygenation. All patients who underwent tracheostomy comprise the cases. The control group comprised all other COVID-19 patients with respiratory failure who did not have a tracheostomy. The Otorhinolaryngology team performed all tracheostomies in operation theaters. We recorded the duration of invasive mechanical ventilation (days) before tracheostomy. We recorded demographics (age, gender, and BMI) and confounding factors to compare the 2 groups. Confounding factors include clinical symptoms and signs on admission. We also recorded laboratory data including white blood cell counts, lymphocyte counts, platelets count, blood chemistries, renal indices, coagulation profile, liver function tests, and inflammatory and disease markers including C-reactive protein, ferritin, procalcitonin, lactate, creatinine kinase, and D-dimers.

Our hospital policy required virus-negative status of the patient to perform tracheostomy. Repeated swab test results recorded seroconversion as per protocol until 2 consecutive swabs were reported as negative. We recorded the number of days to seroconver- sion and the number of swabs performed. Treatment profiles were recorded for use of chloroquine, antivirals, antibacterial, and steroids. All laboratory tests, including PCR confirmation of COVID-19, were performed at the Dubai Hospital Laboratory.

\section{Statistical Analysis}

Sample characteristics were compared between the tracheostomy group and those who did not have tracheostomy. Numerical data were presented as median and interquartile range (IQR), and categorical data were presented as count (\%). Fisher exact tests were performed for categorical variables and the Mann-Whitney $\mathrm{U}$ test for continuous variables (as data were not normally distributed). Data are presented as median and IQR. We used IBM SPSS Statistics for Windows, version 26 (IBM Corp., Armonk, NY, USA).

\section{Results}

We found that tracheostomies were performed only in 21 out of 194 patients (10.8\%). All tracheostomies were performed after 3 weeks ( $22.1 \pm 7.5$ days) on average. Survival was significantly higher in patients who underwent tracheostomy ( 85.7 vs. $42.5 \%, p=0.001$ ). Length of stay in ICU (LOSICU) was longer for tracheostomy patients than controls (median (IQR]: 35 [23-47] vs. 15 [9-21], $p=0.001$ ). Patients who underwent tracheostomy had a higher proportion of treatment with continuous renal replacement therapy (CRRT) (52 vs. $30 \%, p=0.04$ ), more COVID swab testing (6.5 tests [4.5-8.5] vs. 5 [3-7], $p=$ $0.002)$, more days on the ventilator (34.5 days [24-45] vs. 11 [5.5-16.5], $p=0.001)$, more days on mechanical ventilation (34.5 [24-45] vs. 11 [5.5-16.5], $p=0.001)$, and more length of stay in the hospital (54 [38-70] vs. 20 [10.5-29.5], $p=0.001)$. All other factors were not statistically different between the 2 groups (Tables 1-2). Possible false-negative swab test results were $28.8 \%(N=56)$ as swab became positive after being negative (nasal swab = 37 , tracheal swab $=10$, sputum swab $=4$, and nasopharyngeal swab $=5$ ). There was no difference between tracheostomy and no tracheostomy groups. We could not perform long-term temporal follow-up on our patients as the majority of patients transferred to other hospitals, long-term ventilator facilities, or rehabilitation centers owing to relatively limited inpatient beds during COVID-19 pandemic peak time.

\section{Discussion}

Prolonged mechanical ventilation beyond 10 days with a clinical condition requiring more time usually necessitates tracheostomy, which is known to provide comfort, 
Table 1. Sample characteristics (categorical variables)

\begin{tabular}{lcccc}
\hline Categorical variables & Total & Trach & No trach & N value* \\
& $N=194$ & $N=21$ & $N=173$ & 1.0 \\
\hline Gender (male) & $176(90.7)$ & $19(90.4)$ & $157(90.7)$ & 1.0 \\
Test positive-negative-positive test & $56(28.8)$ & $6(28.5)$ & $50(28.9)$ & 0.48 \\
Diabetes & $85(43.81)$ & $11(52.3)$ & $74(42.7)$ & 0.29 \\
Hypertension (yes) & $49(25.2)$ & $3(14.2)$ & $46(26.3)$ & 1.0 \\
Coronary artery disease (yes) & $13(6.7)$ & $1(4.7)$ & $12(6.9)$ & 1.0 \\
Prior renal impairment (yes) & $19(9.7)$ & $2(9.5)$ & $17(9.8)$ & 0.17 \\
Outpatient dialysis (yes) & $14(7.2)$ & $3(14.2)$ & $11(6.3)$ & 1.0 \\
Immunosuppressed (yes) & $8(4.1)$ & $1(4.7)$ & $7(4.0)$ & 1.0 \\
Vasopressors used (yes) & $173(89.1)$ & $19(90.4)$ & $154(89)$ & $52(30)$ \\
Inpatient CRRT (yes) & $63(32.4)$ & $11(52.3)$ & $152(87.8)$ & 0.73 \\
Chloroquine (yes) & $170(87.6)$ & $18(85.7)$ & $68(39.3)$ & 1.0 \\
Lopinavir/ritonavir (yes) & $76(39.1)$ & $8(38)$ & $143(82.6)$ & 1.0 \\
Favipiravir (yes) & $160(82.4)$ & $17(80)$ & $144(83.2)$ & 0.53 \\
Steroids (yes) & $163(84)$ & $19(90)$ & $173(100)$ & 1.0 \\
Sedatives (yes) & $194(100)$ & $21(100)$ & $145(83.8)$ & 0.54 \\
Narcotics (yes) & $164(84.5)$ & $19(90)$ & $164(94.7)$ & 0.61 \\
Paralytics (yes) & $185(95.3)$ & $21(100)$ & $74(42.7)$ & $\mathbf{0 . 0 1}$ \\
Survived (yes) & $92(48.4)$ & $18(85.7)$ & & \\
\hline
\end{tabular}

Values are presented as $n(\%)$. Bold type denotes significance. CRRT, continuous renal replacement therapy. * Fisher's exact test was done to assess the difference between groups.

Table 2. Sample characteristics (continuous variables) and outcome variables

\begin{tabular}{lcccc}
\hline & Total $(N=194)$ & Trach $(N=21)$ & No trach $(N=173)$ & $p$ value* \\
\hline Continuous variables & & & & \\
Age, years & $49(41.5-56.5)$ & $54(46-62)$ & $49(42-56)$ & 0.49 \\
BMI, kg/m & $27.6(24.7-30.4)$ & $27.6(24.7-30.4)$ & $27.6(24.7-30.4)$ & 0.39 \\
Swabs, $n$ & $5(3-7)$ & $6.5(4.5-8.5)$ & $5(3-7)$ & 0.01 \\
WBC, $\times 1,000 / \mu \mathrm{L}$ & $7.4(5.5-9.2)$ & $7.3(5.9-8.7)$ & $7.6(5.5-9.7)$ & 0.67 \\
Ferritin, $\mathrm{ng} / \mathrm{mL}$ & $1,385(682-2,088)$ & $1,080(308-1,851)$ & $1,409(1,196-1,621)$ & 0.45 \\
D-dimer, $\mu \mathrm{g} / \mathrm{mL}$ & $1.2(0-2.45)$ & $0.96(0-2.31)$ & $1.2(0-1.45)$ & 0.53 \\
CRP, mg/L & $125(61.7-188.3)$ & $91.6(60.5-122.7)$ & $132(66.6-197.4)$ & 0.6 \\
Procalcitonin, ng/mL & $0.36(0-1.26)$ & $0.25(0.04-0.45)$ & $0.36(0-0.87)$ & 0.61 \\
Creatinine, mg/dL & $0.9(0.7-1.1)$ & $1(0.82-1.17)$ & $0.9(0.7-1.1)$ & 0.29 \\
Platelets, $\times 1,000 / \mu \mathrm{L}$ & $191(140-241)$ & $208(149-267)$ & $185(135-235)$ & 0.44 \\
pH $(\mathrm{ABG})$ & $7.38(7.31-7.45)$ & $7.32(7.23-7.40)$ & $7.39(7.32-7.45)$ & 0.55 \\
PO, Torr & $63(40.5-85.5)$ & $76(55.6-96.4)$ & $62(38.5-85.5)$ & 0.88 \\
Lactate & $1.6(1-2.2)$ & $1.7(1.2-2.2)$ & $1.6(0.85-2.25)$ & 0.30 \\
Bicarbonate, mmol/L & $22(19.7-24.3)$ & $21.8(20-23.5)$ & $22.2(19.8-24.5)$ & 0.25 \\
PEEP, cm of water & $12(10-14)$ & $10(7.5-12.5)$ & $12(10-14)$ & 0.59 \\
APACHE 2 score & $16(13-21)$ & $18.5(13.5-23.5)$ & $16(12.5-19.5)$ & 0.59 \\
\hline Outcomes & & & \\
Days in ICU, days & $16(9-23)$ & $35(23-47)$ & $15(9-21)$ & 0.01 \\
Days on MV, days & $13(7.5-18.5)$ & $34.5(24-45)$ & $11(5.5-16.5)$ & 0.01 \\
LOS hospital, days & $21(9.5-32.5)$ & $54(38-70)$ & $20(10.5-29.5)$ & 0.01 \\
\hline
\end{tabular}

Values are presented as median (IQR). CRP, C-reactive protein; PEEP, positive-end expiratory pressure; APACHE, Acute Physiology and Chronic Health Evaluation; MV, mechanical ventilation; LOS, length of stay. * Mann-Whitney $\mathrm{U}$ test is used to compare variables. 
reduces amounts of sedatives and paralytic medications, and improves dead space in the respiratory system [5]. Delay in tracheostomy is associated with more sequelae of prolonged sedation and paralysis, neuropathy, and myopathy of critical illness, which may further delay the weaning process [6]. Moreover, prolonged endotracheal intubation requires prolonged invasive indwelling central lines leading to line infection and bacteremia. Prolonged endotracheal intubation frequently requires muscle paralysis leading to increase in the occurrence of pressure injuries and ulcers. We have studied both of these complications in our sample, and data are presently under review (not presented here). Therefore, it is worthwhile to consider tracheostomy in these patients. Aerosol generation during tracheostomy makes it a high-risk procedure; therefore, guidelines suggest that the procedure be delayed until COVID-19 patients have a lower viral burden or become seronegative [7, 8]. Most institutions require 2 reliable negative swabs before tracheostomy is performed since false-negative test results are significantly high [9]. In our sample, $28.8 \%$ of patients had possible false-negative testing as swab again became positive after being negative.

Tracheostomies were performed after 3 weeks in our COVID-19 patients (22.1 \pm 7.5 days). Chao et al. [10] reported the average time of intubation to tracheostomy as $19.7 \pm 6.9$ days, with a range of 8-42 days. In Spanish hospitals, the median timing of tracheostomy was 12 days (4-42 days) since orotracheal intubation [11]. Current protocols recommended delaying tracheostomy for at least 14 days or longer or until a negative PCR $[7,8]$.

Survival was higher in our sample (85\%) for tracheostomy patients. Martin-Villares et al. [11] reported that for $52.1 \%$ of tracheostomized patients, weaning was achieved, while $24.2 \%$ were still under mechanical ventilation, and only $23.7 \%$ patients had died from COVID-19, so their survival was $76.3 \%$. Others reported similarly $67-70 \%$ survival as well $[12,13]$. Weaning details for our sample were unknown as most patients were transferred out of the ICU to another hospital, chronic ventilator-dependent patients' facilities, or rehabilitation centers.

LOSICU was surprisingly prolonged in our tracheostomized patients. Although tracheostomy helps optimally utilize ICU resources, bed crises and cultural and administrative reasons preclude discharging tracheostomized patients to the medical ward.

Days on mechanical ventilation were more in the tracheostomy group. Since more patients survived, they stayed longer on ventilators, ICU, and CRRT. Our region has minimal chronic ventilator-dependent patients' facilities.
We identified the following limitations of our study. The study is a single-center retrospective chart review with a small sample size and low power. Otorhinolaryngologists performed all tracheostomies in the operation theater (no bedside percutaneous tracheostomies are performed). Therefore, results may not apply to other hospitals in the United Arab Emirates or other populations.

\section{Conclusion}

Tracheostomy was performed only in $10 \%$ of our patients with COVID-19 ARDS. Time to tracheostomy was after 3 weeks on average. Survival was better in patients with tracheostomy, but tracheostomized patients stayed longer in the ICU, in the hospital, on mechanical ventilation, and on CRRT.

\section{Acknowledgement}

The authors would like to acknowledge all members of the COVID-19 tracheostomy team who were instrumental in performing tracheostomies (Otorhinolaryngology Department): A.G., I.H., J.K., T.M., K.K., and H.T.

\section{Statement of Ethics}

Ethical approval was provided by DSREC DSREC-07/2020_10/ approved on 23 July 2020. Data source bank was the electronic medical records system (Salama/Epic). Patients' data privacy and confidentiality of information was considered. Only deidentified data were collected. All authors have proper training to exercise strict confidentiality and privacy in data collection. Therefore, strict data security measures were taken to ensure privacy and confidentiality.

\section{Conflict of Interest Statement}

No conflicts to declare.

\section{Funding Sources}

The authors did not receive any funding.

\section{Author Contributions}

R.N.: research idea conception, proposal writing, data collection, data analysis, and manuscript writing. A.N.: idea conception, proposal writing, and reviewing of the final manuscript. M.H., Y.P., N.G., M.H., M.Y., M.M., W.K., M.S., I.M., H.A., A.E., A.W., and A.G.: idea conception and data collection. 


\section{References}

1 WHO Dashboard. 2020 Sep 27. Available from: https://covid19.who.int/.

2 Durbin CG. Indications for and timing of tracheostomy. Respir Care. 2005;50(4):483-7.

3 David AP, Russell MD, El-Sayed IH, Russell MS. Tracheostomy guidelines developed at a large academic medical center during the COVID-19 pandemic. Head Neck. 2020;42(6): 1291-6.

4 Aodeng S, Wang W, Chen Y, Feng G, Wang J, Lv W, et al. Safety and efficacy of tracheotomy for critically ill patients with coronavirus disease 2019 (COVID-19) in Wuhan: a case series of 14 patients. Eur J Cardiothorac Surg. 2020;58(4):745-51.

5 Mattioli F, Fermi M, Ghirelli M, Molteni G, Sgarbi N, Bertellini E, et al. Tracheostomy in the COVID-19 pandemic. Eur Arch Otorhinolaryngol. 2020 Jul;277(7):2133-5.
6 Latronico N, Bolton CF. Critical illness polyneuropathy and myopathy: a major cause of muscle weakness and paralysis. Lancet Neurol. 2011;10(10):931-41.

7 Parker N, Schiff B, Fritz M, Rapaport S, Schild S, Altman K, et al. Tracheotomy recommendations during the COVID-19 pandemic. Am Acad Otorhinolaryngol Head Neck Surg. 2020. Available from: https://www.entnet. org/content/tracheotomy-recommendations-during-covid-19-pandemic.

8 Sommer DD, Engels PT, Weitzel EK, Khalili S, Corsten M, Tewfik MA, et al. Recommendations from the CSO-HNS taskforce on performance of tracheotomy during the COVID-19 pandemic. J Otolaryngol Head Neck Surg. 2020 Apr 27;49(1):273.https://www. entcanada.org/wpcontent/uploads/COVID19-Guidelines-CSOHNS-Task-ForceMar-23-2020.pdf.

9 Fang Y, Zhang H, Xie J, Lin M, Ying L, Pang $\mathrm{P}$, et al. Sensitivity of chest CT for COVID-19: comparison to RT-PCR. Radiology. 2020;296: E115-7.
10 Chao TN, Harbison SP, Braslow BM, Hutchinson CT, Rajasekaran K, Go BC, et al Outcomes after tracheostomy in COVID-19 patients. Ann Surg. 2020;272(3):e181.

11 Martin-Villares C, Molina-Ramirez CP, Bartolome-Benito M, Bernal-Sprekelsen M; COVID ORL ESP Collaberative Group. Outcome of 1890 tracheostomies for critical COVID-19 patients: a national cohort study in Spain. Eur Arch Otorhinolaryngol. 2020:1-8.

12 Abe T, Madotto F, Pham T, Nagata I, Uchida $\mathrm{M}$, Tamiya N, et al. Epidemiology and patterns of tracheostomy practice in patients with acute respiratory distress syndrome in ICUs across 50 countries. Crit Care. 2018; 22(1):195.

13 Young D, Harrison DA, Cuthbertson $\mathrm{BH}$ Rowan K; TracMan Collaborators. Effect of early vs late tracheostomy placement on survival in patients receiving mechanical ventilation: the TracMan randomized trial. JAMA. 2013;309(20):2121-9. 